\title{
Investigation of the association between ten pathogens causing sexually transmitted diseases and high-risk human papilloma virus infection in Shanghai
}

\author{
LI XIE ${ }^{1}$, QIAN LI ${ }^{1}$, XIANGRONG DONG $^{2}$, QI KONG $^{3}$, YUPING DUAN $^{1}$, \\ XIONG CHEN $^{1}$, XIAOQIANG LI ${ }^{1}$, MAO HONG $^{1}$ and TAO LIU ${ }^{1}$ \\ ${ }^{1}$ Clinical Laboratory, Zhongshan Hospital Affiliated to Fudan University Wusong Hospital; \\ ${ }^{2}$ Ear, Nose and Throat Department, Shanghai Gongli Hospital, Shanghai 200940; \\ ${ }^{3}$ School of Biology and Biological Engineering, South China University of \\ Technology, Guangzhou, Guangdong 510641, P.R. China
}

Received July 14, 2020; Accepted March 19, 2021

DOI: $10.3892 /$ mco.2021.2294

\begin{abstract}
Cervical cancer, one of the high-incidence female malignant tumors, has predominated in recent years. Persistent infection with high-risk human papillomavirus (HR-HPV) is the main cause of cervical cancer. Studies have shown that infection with certain sexually transmitted disease (STD) pathogens increases the risk of persistent infection with HR-HPV and is a high-risk factor for cervical cancer. In the present study, cervical specimens were collected for Thinprep cytology test detection, while DNA of cervical cells was extracted for HPV genotyping and detection of 10 STD pathogens, including Neisseria gonorrhoeae, Chlamydia trachomatis (CT), Ureaplasma urealyticum, Ureaplasma urealyticum parvum (Uup)1, Uup3, Uup6, Uup14, Mycoplasma hominis (Mh), Mycoplasma genitalium (Mg) and herpes simplex virus II. Significant differences were observed between CT, Mh and Mg infections and HR-HPV infection $(\mathrm{P}<0.05)$. In addition, CT, Uup3, Uup6 and Mh infections were associated with HR-HPV infection (odds ratio $>1$; $\mathrm{P}<0.05)$. In the comparison of Uup3, Uup6 and Mg infections between the cervical intraepithelial neoplasia (CIN) group
\end{abstract}

Correspondence to: Ms. Qian Li or Ms. Li Xie, Clinical Laboratory, Zhongshan Hospital Affiliated to Fudan University Wusong Hospital, 101 Tong Tai North Road, Shanghai 200940, P.R. China

E-mail: aqianmerry@163.com

E-mail: m18117371006@163.com

Abbreviations:HPV, human papillomavirus; STD, sexually transmitted disease; TCT, Thinprep cytology test; CT, Chlamydia trachomatis; Mh, Mycoplasma hominis; Mg, Mycoplasma genitalium; Uu, Ureaplasma urealyticum; Uup, Ureaplasma urealyticum parvum; NG, Neisseria gonorrhoeae; LSIL, low-grade squamous intraepithelial lesion; HSIL, high-grade squamous intraepithelial lesion; HSV, herpes simplex virus; CIN, cervical intraepithelial neoplasia

Key words: STDs, HPV, TCT, CIN and the control group, statistically significant differences were observed $(\mathrm{P}<0.05)$. In conclusion, the incidences of $\mathrm{CT}, \mathrm{Mh}$ and $\mathrm{Mg}$ infections were similar with HR-HPV infection. CT, Uup6, $\mathrm{Mh}$ and $\mathrm{Mg}$ infections were risk factors for HR-HPV infection. Finally, Uup3, Uup6 and Mg were risk factors of CIN.

\section{Introduction}

Cervical cancer is one type of gynecological malignant tumors with a high incidence rate, which continues to increase year by year. Annually, over 200,000 patients die of cervical cancer worldwide, (1) and $85 \%$ of patients are from developing countries $(1,2)$. Moreover, cervical cancer has predominated in China (3). Cervical cancer can threaten the lives and life quality of women and can impose a heavy burden on the social health system if not addressed promptly and adequately (4).

The female reproductive tract is a complex humid environment that harbors large numbers of microbial organisms that can cause genital disease. However, it remains largely unknown whether these organisms work together to contribute to disease pathogenesis or whether they work against each other. It is also not clear which organisms protect our health and which are detrimental. Among these microbial organisms, persistent high-risk human papilloma virus (HR-HPV) is the main cause of cervical cancer (5). However, cervical cancer does not occur in all patients with HPV; a minority of patients with weak immunity that cannot clear HR-HPV develop persistent infection (6).

A variety of sexually transmitted disease (STD) pathogens are associated with cervical cancer and account for a high proportion of cervical cancer cases, suggesting that STD pathogens play an important role in promoting HR-HPV carcinogenesis $(7,8)$. Studies have shown that infection with certain STD microorganisms may reduce immunity, leading to immune evasion and increasing the risk and severity of HR-HPV infection $(6,9)$. Other pathogens, such as Chlamydia trachomatis (CT), Ureaplasma urealyticum $(\mathrm{Uu})$, and Mycoplasma hominis $(\mathrm{Mh})$, which colonize in the genitourinary tract, can also cause damage, with people 
becoming infected through sexual contact. CT can cause tissue and organ damage, and often co-infects with other STDs (10). Mycoplasma are a genus of bacteria that mainly adhere to the host's susceptible cell receptors through their special surface structure, which damage host cells $(11,12)$. Generally, Uu is divided into two clusters, or 'biovars': Biovar 1/parvo biovar, and biovar 2/T960 (13-15). Biovar 1 consists of four genotypes (1, 3, 6 and 14), while biovar 2 includes 10 serovars $(15,16)$ It is widely accepted that biovar 1 shows fewer signs of danger, while biovar 2 tends to be much more aggressive (17).

Herpes simplex virus II (HSV II) can cause cervical cancer (18). Specifically, HSV-DNA integrates into the DNA of normal tissues, leading to cervical cell lesions (19). Some studies have shown that other STD pathogens, such as Neisseria gonorrhoeae (NG), Uu, Mh, and Mycoplasma genitalium $(\mathrm{Mg})$, can cause repeated infections and change the environment of the genital tract to induce cervical cancer $(20,21)$. The risk of cervical cancer increases with elevation of microbial species in the context of genital tract infection (22).

The complex micro-ecosystem of vagina poses a huge challenge to identifying the true role of each organism. In our study, we found that Ureaplasma urealyticum parvum (Uup6), Uup3, CT, and Mh may contribute to persistent HPV infection, and Uup3, Uup6, and Mg may accelerate cervical intraepithelial neoplasia (CIN) development and thus aggravate HR-HPV-mediated cervical cancer.

\section{Materials and methods}

Methods. This was a retrospective study, ranging from 2012 to 2017 , because of which patients' consent was exempted. A total of 668 patients who underwent gynecological examination were selected from the Department of Gynecology and Gynecology at our hospital from December 2012 to March 2017. The age of patients ranged between 20 and 60 years with a median of 33 years. All patients were subject to the Thinprep cytology test (TCT), while cervical cells were subject to DNA extraction. This study was approved by the relevant ethics committee (acceptance number 2016-Y-02), 11 out of 13 members agreed with the consent exemption.

Cervical cell collection. The cervix was exposed, and cervical secretions were wiped with a speculum by the doctor. A specialized sampling brush, which was used to collect and preserve cervical cells, was inserted into the cervix for five cycles to collect exfoliated epithelial cells from the cervix and cervical canal. The sample brush head was placed in a vial containing a preservative solution and labeled with the identification number of the subject. This was then used to determine the HPV genotype.

$T C T$. A special neck brush was used by the gynecologist to collect exfoliated cells from the outer cervix and cervical canal for five cycles, which were then washed in vials containing a preservative solution. A liquid-based cell smear kit was applied to prepare a uniform thin-layer smear, followed by 95\% alcohol fixation, staining and reading. The 2001 Bethesda System was adopted in the procedure.
Table I. Rates of 668 patients infected with pathogens.

\begin{tabular}{lrr}
\hline Pathogen & Number & $\%$ \\
\hline Human papilloma virus & 415 & 62.13 \\
Neisseria gonorrhoeae & 2 & 0.30 \\
Chlamydia trachomatis & 63 & 9.43 \\
Ureaplasma urealyticum & 79 & 11.83 \\
Uup1 & 66 & 9.88 \\
Uup3 & 128 & 19.16 \\
Uup6 & 105 & 15.72 \\
Uup14 & 2 & 0.30 \\
Mycoplasma hominis & 114 & 17.07 \\
Mycoplasma genitalium & 10 & 1.50 \\
Herpes simplex virus II & 8 & 1.12
\end{tabular}

Uup, Ureaplasma urealyticum parvum.

HPV genotyping. HPV genotyping was performed after polymerase chain reaction amplification using a HPV genotyping kit (Chaozhou Hybribio Biological Technology, China). HPV genotyping flow-through hybridization and gene chip technology (Chaozhou Hybribio Biological Technology) were employed. The chip covered $21 \mathrm{HPV}$ genotypes, including 6, $11,42,43,44,16,18,31,33,35,39,45,51,52,53,56,58,59,66$, 68 and 81. Direct hybridization of DNA fragments on a chip was achieved by observation of colorimetric changes.

Detection of ten STD pathogens. Cervical secretions were tested for 10 pathogens, and flow-through hybridization was used with a nucleic acid detection kit (Chaozhou Hybribio Biological Technology) to identify NG, CT, Uu, Uup1, Uup3, Uup6, Uup14, Mh, Mg and HSV II.

Statistical analysis. Categorical data were compared using the $\chi^{2}$ test or Fisher's exact test to evaluate the relationship between the incidence of HPV and other pathogens, and forward stepwise logistic regression was used for the multivariate analysis. Each of the 10 pathogens was extracted to evaluate the correlation with HPV infection, and then the P-value was decided. $\mathrm{P}<0.05$ was considered statistically significant. Statistical analysis was performed using SPSS 19.0 software.

\section{Results}

Incidence of each pathogen. As listed in Table I, $61 \%$ of patients were infected with HPV. A total of $19.16 \%$ of patients were infected with Uup3, 17.07\% with Mh, 15.72\% with Uup6, $11.83 \%$ with Uu, $9.88 \%$ with Uup1, and $9.43 \%$ with CT. $\mathrm{Mg}$ infection, HSV infection, and NG infection were rare, affecting $1.5,1.12$ and $0.3 \%$ of patient, respectively.

Relationship between HPV infection and other pathogens. In the distribution analysis between HPV infection and infection with other pathogens in the genital tract, there was a significant difference in the distribution of CT, Mh and Mg with HPV infection $(\mathrm{P}<0.05)$, however, the difference between the distribution of other pathogens and HPV infection was not significant (Table II). 
Table II. Association between HPV infection and infection with other sexually transmitted pathogens.

\begin{tabular}{|c|c|c|c|c|c|c|}
\hline Pathogen infection status & Total, $\mathrm{n}$ & $\operatorname{HPV}(-), \mathrm{n}$ & $\operatorname{HPV}(+), n$ & $\operatorname{HPV}(+), \%$ & $\chi^{2}$ & P-value \\
\hline Neisseria gonorrhoeae & & & & & 1.223 & $0.385^{\mathrm{a}}$ \\
\hline Negative & 666 & 253 & 413 & 62.01 & & \\
\hline Positive & 2 & 0 & 2 & 100.00 & & \\
\hline Chlamydia trachomatis & & & & & 5.848 & $0.016^{\mathrm{b}}$ \\
\hline Negative & 605 & 238 & 367 & 60.66 & & \\
\hline Positive & 63 & 15 & 48 & 76.19 & & \\
\hline Ureaplasma urealyticum & & & & & 2.139 & 0.144 \\
\hline Negative & 589 & 229 & 360 & 61.12 & & \\
\hline Positive & 79 & 24 & 55 & 69.62 & & \\
\hline Uup1 & & & & & 0.285 & 0.593 \\
\hline Negative & 602 & 230 & 372 & 61.79 & & \\
\hline Positive & 66 & 23 & 43 & 65.15 & & \\
\hline Uup3 & & & & & 2.953 & 0.086 \\
\hline Negative & 540 & 213 & 327 & 60.56 & & \\
\hline Positive & 128 & 40 & 88 & 68.75 & & \\
\hline Uup6 & & & & & 3.692 & 0.055 \\
\hline Negative & 563 & 222 & 341 & 60.57 & & \\
\hline Positive & 105 & 31 & 74 & 70.48 & & \\
\hline Uup14 & & & & & 0.125 & $0.471^{\mathrm{a}}$ \\
\hline Negative & 666 & 252 & 414 & 62.16 & & \\
\hline Positive & 2 & 1 & 1 & 50.00 & & \\
\hline Mycoplasma hominis & & & & & 20.159 & $<0.001^{\mathrm{b}}$ \\
\hline Negative & 554 & 231 & 323 & 58.30 & & \\
\hline Positive & 114 & 22 & 92 & 80.70 & & \\
\hline Mycoplasma genitalium & & & & & 6.189 & $0.008^{\mathrm{a}, \mathrm{b}}$ \\
\hline Negative & 658 & 253 & 405 & 61.55 & & \\
\hline Positive & 10 & 0 & 10 & 100.00 & & \\
\hline Herpes simplex virus II & & & & & 0.570 & $0.231^{\mathrm{a}}$ \\
\hline Negative & 660 & 251 & 409 & 61.97 & & \\
\hline Positive & 8 & 2 & 6 & 75.00 & & \\
\hline
\end{tabular}

${ }^{a}$ Analyzed using Fisher's exact test. ${ }^{b} \mathrm{P}<0.05$. Uup, Ureaplasma urealyticum parvum; HPV, human papilloma virus.

Logistic regression was used to evaluate the impact of pathogens on HPV infection and other pathogens as in Table III. There was an obvious difference when data were evaluated between HPV infection and CT, Uup3, Uup6, and Mh, indicating that these three pathogens contribute to HPV infection (Table III). In contrast to the results shown in Table I, no significant difference was observed between $\mathrm{Mg}$ infection and HPV infection (P>0.05). NG, Uup1, Uu, Uup14, Mg, and HSVII had no impact on HPV infection $(\mathrm{P}>0.5)$.

Risk analysis of infection and CIN. As shown in Table IV, Uup3, Uup6 and Mg infections increase the risk of CIN. The proportion of patients with Uup3 infection in the CIN group and the control group was $27.27 \%$ (33/121) and $17.37 \%(95 / 547)$, respectively, and the risk of CIN increased in patients with Uup3 infection [odds ratio $(\mathrm{OR})=1.946 ; 95 \%$ confidence interval (CI), 1.200-3.155; $\mathrm{P}=0.007]$. Furthermore, the proportion of patients with Uup6 infection was $20.66 \%(25 / 121)$ and $14.63 \%$ (80/547) in the CIN group and the control group (Table IV), respectively, indicating an increased risk of CIN in patients with Uup6 infection $(\mathrm{OR}=1.712$; 95\% CI 1.009-2.904; $\mathrm{P}=0.046)$. The proportion of patients with $\mathrm{Mg}$ infection was $4.13 \%(5 / 121)$ and $0.91 \%(5 / 547)$ in the CIN group and the control group (Table IV), respectively, suggesting that the risk of CIN was higher in patients with $\mathrm{Mg}$ infection $(\mathrm{OR}=4.207$; 95\% CI, 1.160-15.260; $\mathrm{P}=0.029)$.

Given the variable impact of different pathogens on cervical lesions, cervical lesions were classified. CIN1 was defined as a low-grade squamous intraepithelial lesion (LSIL), while CIN2 and CIN3 were defined as high-grade squamous intraepithelial lesions (HSILs). Patients were more likely to develop cervical cancer with HSILs. Table V shows that among all pathogens, patients who were positive for Uup3, Uup6, or Mh demonstrated low-grade and high-grade 
Table III. Logistic regression analysis of human papilloma virus infection and infection with sexually transmitted pathogens.

\begin{tabular}{|c|c|c|c|c|c|c|c|}
\hline Pathogen & $\begin{array}{l}\text { Regression } \\
\text { coefficient }\end{array}$ & $\begin{array}{l}\text { Standard } \\
\text { deviation }\end{array}$ & Wald & P-value & Odds ratio & $95 \% \mathrm{CI}$ & \\
\hline Neisseria gonorrhoeae & 20.764 & 27838.316 & - & 0.999 & - & 0.000 & - \\
\hline Chlamydia trachomatis & 0.693 & 0.315 & 4.852 & $0.028^{\mathrm{a}}$ & 2.000 & 1.079 & 3.704 \\
\hline Ureaplasma urealyticum & 0.318 & 0.272 & 1.366 & 0.242 & 1.375 & 0.806 & 2.345 \\
\hline Uup1 & 0.254 & 0.286 & 0.789 & 0.374 & 1.289 & 0.736 & 2.257 \\
\hline Uup3 & 0.483 & 0.221 & 4.765 & $0.029^{\mathrm{a}}$ & 1.622 & 1.051 & 2.503 \\
\hline Uup6 & 0.591 & 0.241 & 5.998 & $0.014^{\mathrm{a}}$ & 1.805 & 1.125 & 2.897 \\
\hline Uup14 & -0.246 & 1.430 & 0.034 & 0.853 & 0.767 & 0.047 & 12.645 \\
\hline Mycoplasma hominis & 0.979 & 0.259 & 14.338 & $<0.001^{\mathrm{a}}$ & 2.663 & 1.604 & 4.422 \\
\hline Mycoplasma genitalium & 20.512 & 12184.692 & 0.000 & 0.999 & - & 0.000 & - \\
\hline Herpes simplex virus II & 0.554 & 0.833 & 0.443 & 0.506 & 1.741 & 0.340 & 8.908 \\
\hline
\end{tabular}

${ }^{\mathrm{a}}<0.05$. Uup, Ureaplasma urealyticum parvum.

disease. Patients with LSILs caused by Uup3, Uup6, and Mh accounted for $18.75,15.24$ and $17.54 \%$ of total patients, respectively. Patients with HSILs caused by Uup3, Uup6, and Mh accounted for 7.03, 8.57 and 5.26\% of total patients, respectively (Table V). CT and $\mathrm{Uu}$ accounted for 9.53 and $10.11 \%$ of LSILs, respectively, and 4.76 and $4.50 \%$ of HSILs, respectively (Table V). The number of patients who were positive for other pathogens was too small to evaluate the effect of these pathogens on CIN progression.

\section{Discussion}

HPV infection is a common STD, with an infection rate of approximately $10 \%$ (3) According to data, there are approximately 70 million females with HPV infection in China, and the incidence of cervical cancer in China is approximately 130,000 per year $(1,3)$. There is a certain correlation between infection with different pathogens in the genital tract and cervical cancer and CIN (23). Therefore, cervical cancer researchers are actively searching for synergistic factors of HPV carcinogenesis.

The warm and humid environment of the vagina makes it an ideal habitat for microorganisms. Pathogens, including viruses, bacteria, fungi, and mycoplasma, can easily breed. Many of these organisms can cause disease in the reproductive tract. Until now, little has been known about the distribution of pathogenic species in the vaginal and their exact role in cervical disease formation. In this study, HPV and 10 other pathogens were evaluated. The incidence of HPV was $61 \%$. Approximately $19.16 \%$ of patients were affected by Uup3, $17.07 \%$ with Mh, $15.72 \%$ with Uup6, $11.83 \%$ with Uu, $9.88 \%$ with Uup1, and $9.43 \%$ with CT. In line with previous study, (24) Uu, Uup1, Uup3, Uup6, and Uup14 were most frequently observed.

Furthermore, CT, Mh, and Mg shared similar infection rates with HR-HPV (Table II; P<0.05). Possible synergistic effects also existed between CT, Uup3, Uup6, and Mh infections and HR-HPV infection (Table III). CT, Uup3, Uup6, and Mh likely contribute to HPV infection (25), which was different from the species that contributed to CIN progression.
Combined with the results presented in Table IV, we note that it is not the exact same species of pathogen with that in Table III, that could promote CIN progression though mostly similar. CT, Uup3, Uup6, and Mg played an obvious role in CIN formation and progression. However, in patients infected with multiple pathogens and who harbored HR-HPV infection, it was not clear if it was the latter that was largely responsible for CIN formation and progression, especially in patients with HSILs.

Table $\mathrm{V}$ shows that a large proportion of patients with Uup3, Uup6, and Mh had HSILs and LSILs. Patients infected with Uup3, Uup6, and Mh with LSILs accounted for 18.75, 15.24 and $17.54 \%$ of total patients, respectively. Patients infected with Uup3, Uup6, and Mh with HSILs accounted for $7.03,8.57$ and $5.26 \%$ of total patients, respectively (Table V). Patients with HSILs were much more likely to develop cervical cancer. Thus, certain species of Uu are hazardous, including Uup3and Uup6. However, the role of HR-HPV in HSIL formation is still unknown, because not a small part of patients shares multi-pathogen coinfection (26). This could be because different HPV genotypes have synergistic effects on each other (7) or because different pathogens promote HPV persistence. More data will be required to differentiate and distinguish the role of each of these pathogens.

Our results show that HR-HPV infection increases the risk of $\mathrm{CT}$ infection, but there was no significant correlation between $\mathrm{CT}$ infection and CIN, possibly due to the small sample size, which may have failed to detect a sufficient number of positive patients. It was speculated that CT could increase the risk of CIN by synergistic action with HPV. No relationship was identified between $\mathrm{Mh}$ and $\mathrm{CIN}$, but $\mathrm{Mh}$ did have a positive effect on HPV persistence.

It is believed that $\mathrm{Uu}$ is related to the persistence of HPV infection and early cervical cytological changes (27). The rate of Uu infection increases in HPV-positive patients and in patients with cervical cancer, and the increase in $\mathrm{Uu}$ infection is a significantly correlated with the occurrence of CIN caused by HPV infection $(26,28)$; therefore, it should be highly valued when Uu infection is combined with HPV infection (28), which was frequently observed in our study. 
Table IV. Pathogens and risk analysis of cervical lesions.

\begin{tabular}{|c|c|c|c|c|}
\hline Pathogen infection status & Control, n (\%) & Intraepithelial lesion, $\mathrm{n}(\%)$ & Odds ratio $(95 \% \mathrm{CI})$ & P-value \\
\hline \multicolumn{5}{|l|}{ Neisseria gonorrhoeae } \\
\hline Negative & $545(99.63)$ & $121(100.00)$ & 1 & \\
\hline Positive & $2(0.37)$ & $0(0.00)$ & - & - \\
\hline \multicolumn{5}{|l|}{ Chlamydia trachomatis } \\
\hline Negative & $493(90.13)$ & $112(92.56)$ & 1 & \\
\hline Positive & $54(9.87)$ & $9(7.44)$ & $0.706(0.334-1.493)$ & 0.361 \\
\hline \multicolumn{5}{|l|}{ Ureaplasma urealyticum } \\
\hline Negative & $481(87.93)$ & $108(89.26)$ & 1 & \\
\hline Positive & $66(12.07)$ & $13(10.74)$ & $0.894(0.462-1.731)$ & 0.741 \\
\hline \multicolumn{5}{|l|}{ Uup1 } \\
\hline Negative & $491(89.76)$ & $111(91.74)$ & 1 & \\
\hline Positive & $56(10.24)$ & $10(8.26)$ & $0.944(0.455-1.959)$ & 0.877 \\
\hline \multicolumn{5}{|l|}{ Uup3 } \\
\hline Negative & $452(82.63)$ & $88(72.73)$ & 1 & \\
\hline Positive & $95(17.37)$ & $33(27.27)$ & $1.946(1.200-3.155)$ & $0.007^{\mathrm{a}}$ \\
\hline \multicolumn{5}{|l|}{ Uup6 } \\
\hline Negative & $467(85.37)$ & $96(79.34)$ & 1 & \\
\hline Positive & $80(14.63)$ & $25(20.66)$ & $1.712(1.009-2.904)$ & $0.046^{\mathrm{a}}$ \\
\hline \multicolumn{5}{|l|}{ Uup14 } \\
\hline Negative & $545(99.63)$ & $121(100.00)$ & 1 & \\
\hline Positive & $2(0.37)$ & $0(0.00)$ & - & - \\
\hline \multicolumn{5}{|l|}{ Mycoplasma hominis } \\
\hline Negative & $459(83.91)$ & $95(78.51)$ & 1 & \\
\hline Positive & $88(16.09)$ & $26(21.49)$ & $1.358(0.812-2.271)$ & 0.244 \\
\hline \multicolumn{5}{|l|}{ Mycoplasma genitalium } \\
\hline Negative & $542(99.09)$ & $116(95.87)$ & 1 & \\
\hline Positive & $5(0.91)$ & $5(4.13)$ & $4.207(1.160-15.260)$ & $0.029^{\mathrm{a}}$ \\
\hline \multicolumn{5}{|l|}{ Herpes simplex virus II } \\
\hline Negative & $541(98.90)$ & $119(98.35)$ & 1 & \\
\hline Positive & $6(1.10)$ & $2(1.65)$ & $1.618(0.313-8.346)$ & 0.566 \\
\hline
\end{tabular}

${ }^{\mathrm{a}} \mathrm{P}<0.05$. Uup, Ureaplasma urealyticum parvum.

Table V. Human papilloma virus and risk analysis of cervical lesions.

\begin{tabular}{lccc}
\hline Pathogen & Positive, $\mathrm{n}$ & LSIL, n $(\%)$ & HSIL, $\mathrm{n}(\%)$ \\
\hline Neisseria gonorrhoeae & 2 & $0(0.00)$ & $0(0.00)$ \\
Chlamydia trachomatis & 63 & $6(9.53)$ & $3(4.76)$ \\
Ureaplasma urealyticum & 89 & $9(10.11)$ & $4(4.50)$ \\
Uup1 & 76 & $9(11.84)$ & $1(1.31)$ \\
Uup3 & 128 & $24(18.75)$ & $9(7.03)$ \\
Uup6 & 105 & $16(15.24)$ & $9(8.57)$ \\
Uup14 & 2 & $0(0.00)$ & $0(0.00)$ \\
Mycoplasma hominis & 114 & $20(17.54)$ & $6(5.26)$ \\
Mycoplasma genitalium & 10 & $2(20.00)$ & $3(30.00)$ \\
Herpes simplex virus II & 8 & $0(0.00)$ & $2(25.00)$ \\
\hline
\end{tabular}

Uup, Ureaplasma urealyticum parvum; LSIL, low-grade squamous intraepithelial lesion; HSIL, high-grade squamous intraepithelial lesion. 
HSV II infection increases the risk of cervical cancer and has a synergistic effect with HPV (29). It has also been confirmed that HSV DNA integrates into the DNA of normal cells to promote cancer cell development (30). However, HSV II infection is mostly asymptomatic. The results of this study show that HSV II infection is associated with HR-HPV infection, but not with the CIN development. This can be ascribed to regional differences and low positive infection rates.

In conclusion, the incidence of cervical cancer is complex and is determined by a variety of factors. Multiple STD pathogens are involved in the process of HR-HPV carcinogenesis. Varying conclusions have been drawn worldwide, which may be related to differences in the prevalence and methods used to detect STD pathogens between regions. Overall, we show that CT, Uup3, Uup6, and Mh could be risk factors for HR-HPV persistence and that Uup6, Uup4, and Mg significantly impact CIN progression.

\section{Acknowledgements}

The authors would like to thank Dr Xiaoqiang Li and Mr. Xiong Chen (both Wusong Hospital, Shanghai, China) for their help with the experiments.

\section{Funding}

No funding was received.

\section{Availability of data and materials}

The datasets generated and/or analyzed during the current study are available in the Baidu Cloud repository (https://pan.baidu. com/s/1Q566BTCHCq7sEfUkkIsGwQ; reference no. w1fa).

\section{Authors' contributions}

LX and QL conceived, designed and performed the experiments, analyzed the data and drafted the manuscript. XL provided the samples, helped design the experiments and contributed to data analysis. XD, QL, MH and YD performed the experiments. QK, TL and XC helped to analyze the data and performed the experiments. LX and TL contributed to reagents/materials/analysis tools. LX and QL confirmed the authenticity of the raw data. All authors read and approved the final manuscript.

\section{Ethics approval and consent to participate}

The present study was approved by the Ethics Committee of Zhongshan Hospital Affiliated to Fudan University Wusong Hospital (approval no. 2016-Y-02; Shanghai, China). Patient consent was waived due to the retrospective nature of the study.

\section{Patient consent for publication}

Not applicable.

\section{Competing interests}

The authors declare that they have no competing interests.

\section{References}

1. Venkatas J and Singh M: Cervical cancer: A meta-analysis, therapy and future of nanomedicine. Ecancermedicalscience 14: $1111,2020$.

2. Bray F, Ferlay J, Soerjomataram I, Siegel RL, Torre LA and Jemal A: Global cancer statistics 2018: GLOBOCAN estimates of incidence and mortality worldwide for 36 cancers in 185 countries. CA Cancer J Clin 68: 394-424, 2018.

3. Zhang J, Cheng K and Wang Z: Prevalence and distribution of human papillomavirus genotypes in cervical intraepithelial neoplasia in China: A meta-analysis. Arch Gynecol Obstet 302: 1329-1337, 2020.

4. Chesson HW, Meites E, Ekwueme DU, Saraiya M and Markowitz LE: Updated medical care cost estimates for HPV-associated cancers: Implications for cost-effectiveness analyses of HPV vaccination in the United States. Hum Vaccin Immunother 15: 1942-1948, 2019.

5. Schwarz E, Freese UK, Gissmann L, Mayer W, Roggenbuck B Stremlau A and zur Hausen H: Structure and transcription of human papillomavirus sequences in cervical carcinoma cells. Nature 314: 111-114, 1985.

6. Galliverti G, Wullschleger S, Tichet M, Murugan D, Zangger N, Horton W, Korman AJ, Coussens LM, Swartz MA and Hanahan D: Myeloid cells orchestrate systemic immunosuppression, impairing the efficacy of immunotherapy against $\mathrm{HPV}^{+}$ cancers. Cancer Immunol Res 8: 131-145, 2020.

7. Hajia M and Sohrabi A: Possible synergistic interactions among multiple HPV genotypes in women suffering from genital neoplasia. Asian Pac J Cancer Prev 19: 785-789, 2018.

8. Looker KJ, Rönn MM, Brock PM, Brisson M, Drolet M, Mayaud P and Boily MC: Evidence of synergistic relationships between HIV and human papillomavirus (HPV): Systematic reviews and meta-analyses of longitudinal studies of HPV acquisition and clearance by HIV status, and of HIV acquisition by HPV status. J Int AIDS Soc 21: e25110, 2018.

9. Senba M and Mori N: Mechanisms of virus immune evasion lead to development from chronic inflammation to cancer formation associated with human papillomavirus infection. Oncol Rev 6: e17, 2012.

10. Liang Y, Chen M, Qin L, Wan B and Wang H: A meta-analysis of the relationship between vaginal microecology, human papillomavirus infection and cervical intraepithelial neoplasia. Infect Agent Cancer 14: 29, 2019.

11. Kamminga T, Koehorst JJ, Vermeij P, Slagman SJ, Martins Dos Santos VA, Bijlsma JJ and Schaap PJ: Persistence of functional protein domains in mycoplasma species and their role in host specificity and synthetic minimal life. Front Cell Infect Microbiol 7: 31, 2017.

12. Schnee C, Schulsse S, Hotzel H, Ayling RD, Nicholas RA, Schubert E, Heller M, Ehricht R and Sachse K: A novel rapid DNA microarray assay enables identification of 37 mycoplasma species and highlights multiple mycoplasma infections. PLoS One 7: e33237, 2012.

13. Nelson S, Matlow A, Johnson G, Th'ng C, Dunn M and Quinn P: Detection of Ureaplasma urealyticum in endotracheal tube aspirates from neonates by PCR. J Clin Microbiol 36: 1236-1239, 1998.

14. Grattard F, Pozzetto B, de Barbeyrac B, Renaudin H, Clerc M, Gaudin OG and Bébéar C: Arbitrarily-primed PCR confirms the differentiation of strains of Ureaplasma urealyticum into two biovars. Mol Cell Probes 9: 383-389, 1995.

15. Kong F, Zhu X, Wang W, Zhou X, Gordon S and Gilbert GL: Comparative analysis and serovar-specific identification of multiple-banded antigen genes of Ureaplasma urealyticum biovar 1. J Clin Microbiol 37: 538-543, 1999.

16. Ruifu Y, Minli Z, Guo Z and Wang X: Biovar diversity is reflected by variations of genes encoding urease of Ureaplasma urealyticum. Microbiol Immunol 41: 625-627, 1997.

17. Deguchi T, Yasuda M and Maeda S: Non-chlamydial nongonococcal urethritis. Nihon Rinsho 67: 167-171, 2009 (In Japanese).

18. Worboys M: In The Hidden Affliction: Sexually Transmitted Infections and Infertility in History. Szreter S (ed). University of Rochester Press, Rochester, NY, 2019.

19. Kit S, Hazen M, Otsuka H, Qavi H, Trkula D and Dubbs DR: The site of integration of the herpes simplex virus type 1 thymidine kinase gene in human cells transformed by an HSV-1 DNA fragment. Int J Cancer 28: 767-776, 1981. 
20. Horner P, Donders G, Cusini M, Gomberg M, Jensen JS and Unemo M: Should we be testing for urogenital Mycoplasma hominis, Ureaplasma parvum and Ureaplasma urealyticum in men and women?-a position statement from the European STI Guidelines Editorial Board. J Eur Acad Dermatol Venereol 32: 1845-1851, 2018.

21. Hlatshwayo M, Reno HEL and Yarbrough ML: STI update: Testing, treatment, and emerging threats. Cleve Clin J Med 86: 733-740, 2019.

22. Oh HY, Kim BS, Seo SS, Kong JS, Lee JK, Park SY, Hong KM Kim HK and Kim MK: The association of uterine cervical microbiota with an increased risk for cervical intraepithelial neoplasia in Korea. Clin Microbiol Infect 21: 674.e1-9, 2015.

23. Hakama M, Luostarinen T, Hallmans G, Jellum E, Koskela P, Lehtinen M, Thoresen S, Youngman L and Hakulinen T: Joint effect of HPV16 with Chlamydia trachomatis and smoking on risk of cervical cancer: Antagonism or misclassification (Nordic countries). Cancer Causes Control 11: 783-790, 2000.

24. Kletzel HH, Rotem R, Barg M, Michaeli J and Reichman O: Ureaplasma urealyticum: The role as a pathogen in women's health, a systematic review. Curr Infect Dis Rep 20: 33, 2018.

25. Wang L, Zhu L, Li H, Ma N, Huang H, Zhang X, Li Y and Fang J: Association between asymptomatic sexually transmitted infections and high-risk human papillomavirus in cervical lesions. J Int Med Res 47: 5548-5559, 2019.

26. Verteramo R, Pierangeli A, Mancini E, Calzolari E, Bucci M, Osborn J, Nicosia R, Chiarini F, Antonelli G and Degener AM: Human Papillomaviruses and genital co-infections in gynaecological outpatients. BMC Infect Dis 9: 16, 2009.
27. Lukic A, Canzio C, Patella A, Giovagnoli M, Cipriani P, Frega A and Moscarini M: Determination of cervicovaginal microorganisms in women with abnormal cervical cytology: The role of ureaplasma urealyticum. Anticancer Res 26: 4843-4849, 2006.

28. Biernat-Sudolska M, Szostek S, Rojek-Zakrzewska D, Klimek M and Kosz-Vnenchak M: Concomitant infections with human papillomavirus and various mycoplasma and ureaplasma species in women with abnormal cervical cytology. Adv Med Sci 56: 299-303, 2011.

29. Smith JS, Herrero R, Bosetti C, Muñoz N, Bosch FX, Eluf-Neto J, Castellsagué X, Meijer CJ, Van den Brule AJ, Franceschi S, et al: Herpes simplex virus-2 as a human papillomavirus cofactor in the etiology of invasive cervical cancer. J Natl Cancer Inst 94: 1604-1613, 2002

30. Li L, Zhu Q and Zheng S: Detection of human papillomavirus and herpesvirus genotypes in biopsy specimens from cervical carcinoma by PCR-endonuclease cleavage. Zhonghua Shi Yan He Lin Chuang Bing Du Xue Za Zhi 13: 235-238, 1999 (In Chinese).

(i) $\Theta$ This work is licensed under a Creative Commons Attribution-NonCommercial-NoDerivatives 4.0 International (CC BY-NC-ND 4.0) License. 